Schmerz 2017 $\cdot 31: 383-390$

DOI 10.1007/s00482-016-0184-8

Online publiziert: 11. Januar 2017

(c) Der/die Autor(en) 2016. Dieser Artikel ist

eine Open-Access-Publikation.

CrossMark

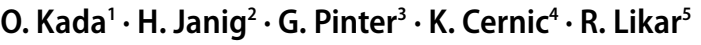

'Studiengang Gesundheits- und Pflegemanagement, FH Kärnten, Feldkirchen, Österreich

${ }^{2}$ Institut für Psychologie, Alpen-Adria Universität Klagenfurt, Klagenfurt, Österreich

${ }^{3}$ Abteilung für Akutgeriatrie und Remobilisation, Klinikum Klagenfurt, Klagenfurt, Österreich

${ }^{4}$ Kaufmännische Direktion, Klinikum Klagenfurt, Klagenfurt, Österreich

${ }^{5}$ Abteilung für Anästhesie und Intensivmedizin, Zentrum für interdisziplinäre Schmerztherapie und

Palliativmedizin, Klinikum Klagenfurt, Klagenfurt, Österreich

\section{Palliativversorgung in Pflegeheimen}

\section{Ergebnisse einer Befragung zu Wissen und Selbstwirksamkeitserwartung von Pflegepersonal}

\section{Hintergrund und Ausgangslage}

„Palliativmedizin - auch im hohen Alter?" titelten Sandgathe Husebø u. Husebø [24] vor 15 Jahren und führten zahlreiche Argumente ins Feld, die die Bedeutung von Palliative Care im Pflegeheim unterstreichen und bis heute nicht an Gültigkeit verloren haben: Pflegeheimbewohner sind zumeist komplex krank [20], viele von ihnen leiden an Demenz und können Schmerzen nur schwer artikulieren [19], weshalb Bewohner mit mittelgradiger bis schwerer kognitiver Beeinträchtigung ein erhöhtes Risiko einer schmerztherapeutischen Unterversorgung aufweisen [3]. Wissenslücken in Palliative Care bei Pflegekräften und Hausärzten (z. B. [13, 18, 23]) und deren wenig kontinuierliche Zusammenarbeit erschweren eine adäquate Schmerztherapie. Ergebnisse der SHELTER-Studie (Services and Health for Elderly in Long-Term Care [15]), die 3926 Pflegeheimbewohner aus 7 EU-Ländern (u. a. Deutschland) einschloss, deuten darauf hin, dass Defizite in der Diagnostik und Therapie von Schmerzen nach wie vor bestehen; das Schmerzmanagement von 20 bis $35 \%$ der Pflegeheimbewohner ist als insuffizient einzustufen.

Das österreichische Konzept der Palliativversorgung ist europaweit beispielgebend und erfolgt idealerweise in einem abgestuften System [9]. Für jede Art der Grundversorgung (Krankenhäuser; Alten- und Pflegeheime; familiärer Bereich - zu Hause) gibt es sowohl unterstützende als auch betreuende Angebote. Im Krankenhausbereich sind es die Palliativkonsiliardienste bzw. Palliativstationen; in der Langzeitpflege die Hospizteams und mobilen Palliativteams bzw. stationäre Hospize; im familiären Bereich die mobilen Palliativteams bzw. Tageshospize [9]. In Kärnten kommt den mobilen Palliativteams bestehend aus Ärzten und Pflegekräften sowie Psychologen und Sozialarbeitern eine zentrale Bedeutung in der Palliativversorgung von Pflegeheimbewohnern zu [25]. Hospiz Österreich [10] hat Richtlinien entwickelt, wie über einen Organisationsentwicklungsprozess Hospiz und Palliative Care (HPC) in Alten- und Pflegeheimen integriert werden soll; entsprechende Modellprojekte wurden bereits umgesetzt, wobei im Bundesland Kärnten derzeit ein Modellprojekt läuft. Einer österreichweiten Bestandsaufnahme zu Hospiz- und Palliative Care in Alten- und Pflegeheimen $(N=284,37$ \% Ausschöpfung) zufolge gibt es neben den Modellprojekten auch weitere Heime, die sich um die Integration von HPC bemühen [14]. Gleichzeitig muss aber festgehalten werden, dass noch viel Optimierungspotenzial gegeben ist. Beispielswei- se wird bislang nur in wenigen Heimen mit Palliativmedizinern zusammengearbeitet; so verfügen $6 \%$ der Heime über einen fest angestellten Palliativmediziner und $18 \%$ über einen Palliativmediziner auf Konsiliarbasis [14]. Entsprechendes Wissen und Kompetenzen insbesondere beim Pflegepersonal, aber auch bei Hausärzten und Notärzten, sind eine weitere zentrale Voraussetzung für eine erfolgreiche Implementierung von HPC im Pflegeheimbereich, die noch nicht ausreichend vorhanden zu sein scheint [11]. Inwiefern diese Voraussetzungen aufseiten des Pflegepersonals erfüllt sind, soll mit der vorliegenden Studie untersucht werden. Entsprechend wurden der Wissensstand und die spezifische Selbstwirksamkeitserwartung (SWE) von Pflegekräften in österreichischen Alten- und Pflegeheimen (Bundesland Kärnten) erhoben. Klinisches Fachwissen allein führt noch nicht zu entsprechendem Verhalten [22], zusätzlich muss auch die Überzeugung über die eigene Kompetenz gegeben sein (SWE). Wie von Bandura in seiner sozial-kognitiven Theorie postuliert, ist die Ausprägung der SWE eine Erklärung dafür, dass Personen trotz gleichen Wissens oder gleicher Fähigkeiten unterschiedlich mit Herausforderungen und Stressoren umgehen: „Perceived self-efficacy is not a measure of the skills one has but a belief about what one can do under dif- 


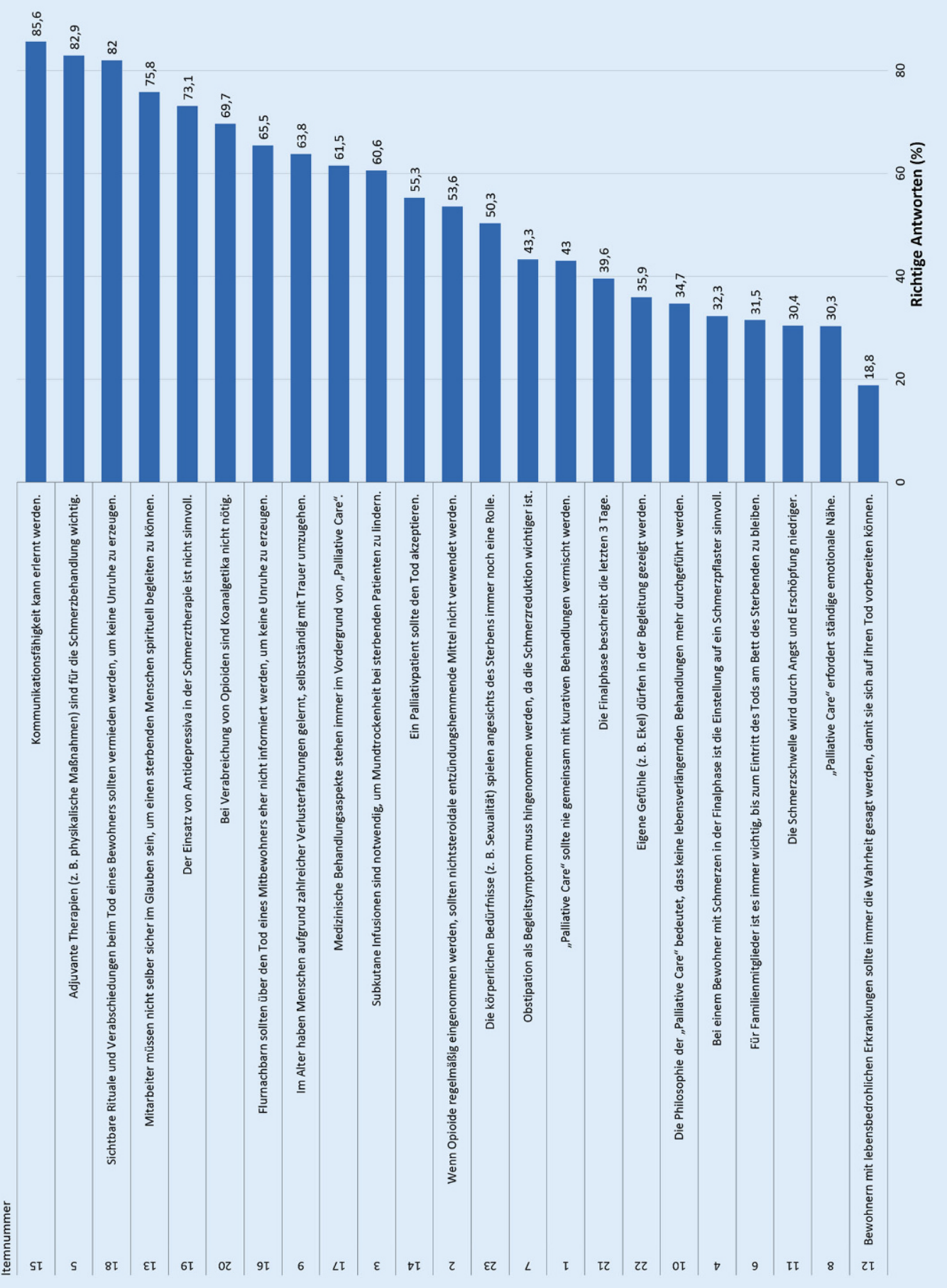

Abb. $1 \triangleleft$ Anteil richtig beantworteter Fragen der BPW-Wissensskala auf Einzelitemebene. Anmerkung: Items 1, 2, 3, 4, 6, 7, 8, 9, 10, $12,14,16,17,18,19,20$ sind negativ gepolt (Ablehnung richtige Antwort)

ferent sets of conditions with whatever skills one possesses“ [1, S. 37]. Dabei ist jeweils die spezifische SWE ein besserer Prädiktor des Verhaltens als die allgemeine, da sich die SWE in Bezug auf verschiedene Lebensbereiche und Verhaltensweisen durchaus unterscheiden kann $[1,2]$. In der vorliegenden Studie steht folglich die SWE bezogen auf den Umgang mit Palliativpatienten im Pflegeheim im Zentrum.

Effekte der Berufsgruppe (diplomiertes vs. nichtdiplomiertes Pflegepersonal) und der Berufserfahrung in der Geriatrie (weniger als 5 Jahre, 5-10 Jahre, mehr als 10 Jahre) wurden getestet. Aufgrund der deutlich längeren Ausbildung von diplomiertem Pflegepersonal würde man einen Wissensvorsprung und auch eine höhere SWE gegenüber nichtdiplomiertem Personal erwarten. Mit zunehmender Berufserfahrung sollte die SWE steigen, zumal direkte Erfahrungen eigener Kompetenz die stärkste Quelle der SWE 
darstellen [1, 2]. Darüber hinaus sollte der Zusammenhang zwischen Wissen und SWE geprüft werden.

\section{Material und Methoden}

\section{Studiendesign und Methodik der Datenerhebung}

Im Zuge der querschnittlich angelegten Untersuchung wurde Pflegepersonal aus 17 Pflegeheimen im Bundesland Kärnten unter kontrollierten Bedingungen im Zuge von Teamsitzungen und unter Testleitung einer geschulten Projektmitarbeiterin (von der Erstautorin geschulte Gesundheits- und Pflegemanagementstudentin) befragt. Die Teilnahme an der Befragung war freiwillig und die ausgefüllten Fragebögen konnten anonymisiert in vorbereitete Antwortboxen eingeworfen werden. Zum Einsatz kam dabei der Bonner Palliativwissenstest (BPW), der von Pfister et al. [22] speziell für Pflegeheimpersonal entwickelt wurde. Dieser Fragebogen umfasst 23 positiv und negativ gepolte Wissensfragen zum Thema Palliativversorgung im Pflegeheim (Wissensskala), die durch Addition der korrekten Antworten zu einem Skalenwert aufsummiert werden (möglicher Wertebereich 0 bis 23). Die Testautoren berichten eine interne Konsistenz von Cronbachs $\alpha=0,71$, in der vorliegenden Studie lag diese bei nur 0,53. Des Weiteren umfasst der BPW 15 Fragen mit 4-stufigem Antwortformat (stimmt nicht, stimmt kaum, stimmt eher, stimmt) zur Messung der SWE in Bezug auf die Palliativversorgung von Pflegeheimbewohnern (SWESkala). Über Mittelwertsbildung wurde der Skalenwert der SWE-Skala gebildet, wodurch sich ein Wertebereich zwischen 1 und 4 ergibt. Es wird eine zufriedenstellende interne Konsistenz berichtet (Cronbachs $\alpha=0,86$ ), die auch in der vorliegenden Studie bestätigt wurde (Cronbachs $\alpha=0,84$ ). Außerdem wurden die Berufsgruppe (diplomiertes vs. nichtdiplomiertes Pflegepersonal) sowie die Berufserfahrung in der Altenpflege erfasst (weniger als 5 Jahre, 5-10 Jahre, mehr als 10 Jahre). Auf die Erhebung weiterer soziodemografischer Daten wurde

Schmerz 2017-31:383-390 DOI 10.1007/s00482-016-0184-8

(c) Der/die Autor(en) 2016. Dieser Artikel ist eine Open-Access-Publikation.

O. Kada $\cdot$ H. Janig $\cdot$ G. Pinter $\cdot$ K. Cernic $\cdot$ R. Likar Palliativversorgung in Pflegeheimen. Ergebnisse einer Befragung
zu Wissen und Selbstwirksamkeitserwartung von Pflegepersonal

\section{Zusammenfassung}

Hintergrund. Die zunehmende Konfrontation mit Palliativpatienten in Altenund Pflegeheimen stellt das betreuende pflegerische und ärztliche Personal vor große Herausforderungen. Defizite in der palliativen Versorgung geriatrischer Patienten wurden wiederholt nachgewiesen, viele Pflegeheimbewohner - insbesondere jene mit Demenz - sind schmerztherapeutisch unterversorgt.

Ziel der Arbeit. In der vorliegenden Studie wurden Wissen und Selbstwirksamkeitserwartung (SWE) von Pflegepersonal im Bundesland Kärnten (Österreich) in Bezug auf Palliative Care von Pflegeheimbewohnern erhoben.

Material und Methoden. $N=330$ Pflegepersonen wurden mithilfe des Bonner Palliativwissenstests (BPW, Pfister et al., 2011) befragt, der Wissen und SWE von Pflegepersonal in Alten- und Pflegeheimen erfasst. Neben einer deskriptivstatistischen Auswertung wurden Effekte der Berufsgruppe (diplomiert vs. nichtdiplomiert) und der Berufserfahrung analysiert.

Ergebnisse. Im Durchschnitt wurde etwas mehr als die Hälfte der Wissensfragen korrekt beantwortet. Die SWE war insgesamt sehr hoch ausgeprägt. Diplomierte Pflegepersonen wiesen ein höheres Wissen und eine höhere SWE auf als nichtdiplomierte Pflegepersonen. Effekte der Berufserfahrung zeigten sich lediglich im Hinblick auf die SWE. Schlussfolgerung. Die Ergebnisse sind gut vergleichbar mit Befunden aus Deutschland und weisen auf die Notwendigkeit von Interventionen zur Wissensvermittlung als wichtige Grundlage einer angemessenen Palliativversorgung für Pflegeheimbewohner hin.

Schlüsselwörter

Palliative Care · Pflegeheim · Wissen . Selbstwirksamkeitserwartung · Palliative Gerontologie

\section{Palliative care in nursing homes. Results of a survey about knowledge and self-efficacy of nursing staff}

\section{Abstract}

Background. Nursing homes are confronted more and more with palliative care patients, which present a challenge for nursing and medical personnel. Deficits in the palliative care of geriatric patients have been repeatedly demonstrated and many nursing home residents, especially those suffering from dementia, are undersupplied regarding pain management.

Objectives. The present study was carried out to measure the knowledge and self-efficacy of nursing staff in the province of Carinthia (Austria) regarding palliative care of nursing home residents.

Material and methods. A total of 330 nursing personnel were surveyed using the Bonn test for knowledge in palliative care (BPW), which measures knowledge and self-efficacy in nursing home personnel. In addition to descriptive analyses, the effects of the professional group (registered nurses vs. nursing assistants) and working experience were tested.

Results. On average a little more than half of the knowledge items were answered correctly. Nurses' self-efficacy was high. Registered nurses exhibited more knowledge and higher self-efficacy compared to nursing assistants. Effects of working experience could only be demonstrated regarding selfefficacy.

Conclusion. The results are to a large extent in line with results from Germany and indicate the necessity of interventions for improving nurses' knowledge as a major basis for adequate palliative care in nursing home residents.

Keywords

Palliative care - Nursing home - Knowledge . Self-efficacy · Palliative gerontology 


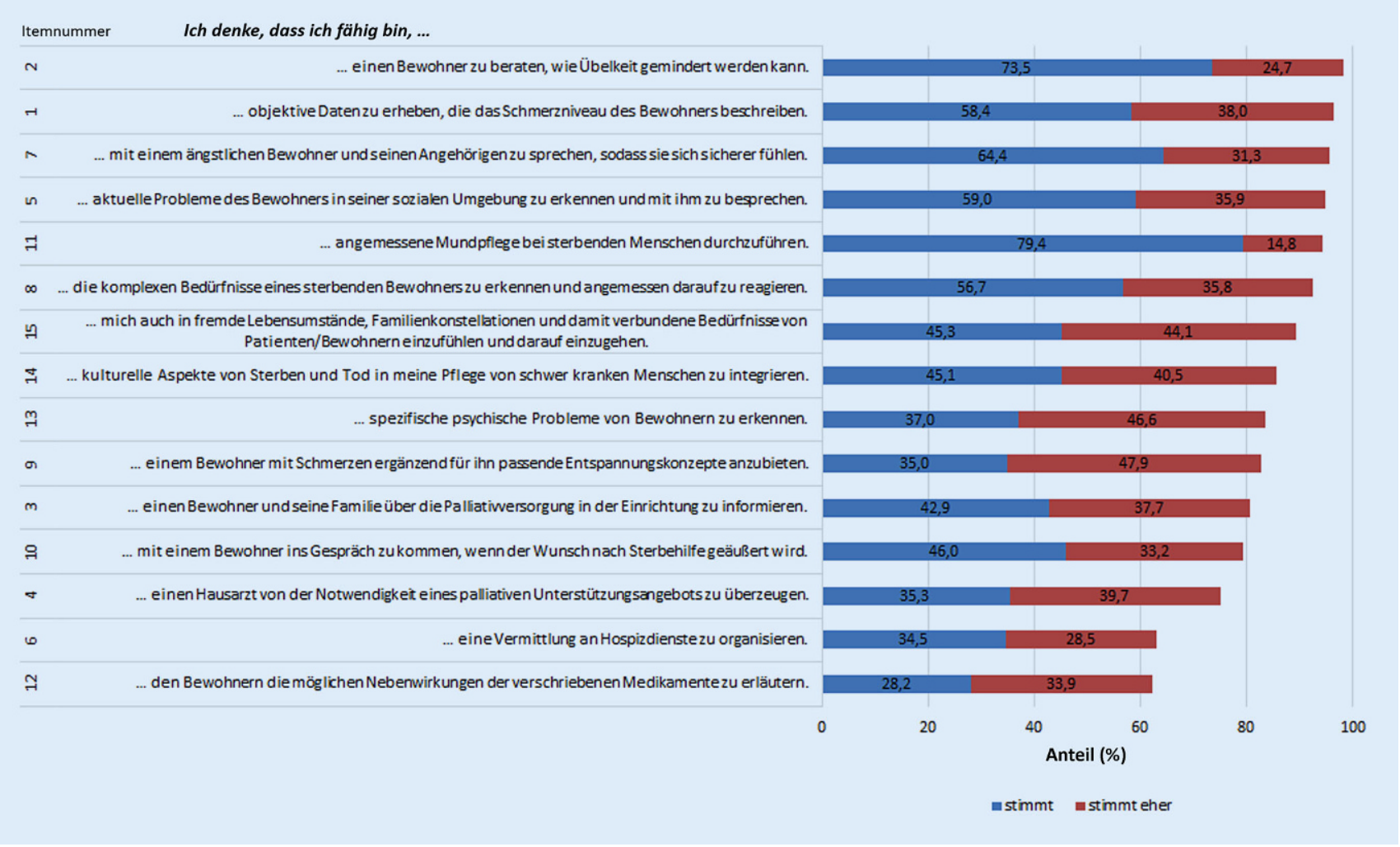

Abb. 2 A Auswertung der Selbstwirksamkeitserwartungsskala (SWE) des Bonner Palliativwissenstests (BPW) auf Einzelitemebene

zugunsten einer hohen Rücklaufquote verzichtet.

\section{Stichprobe}

Es liegen auswertbare Daten von 330 Pflegepersonen vor, was gemessen am Personalstand der 17 Heime einer Ausschöpfungsquote von $61 \%$ entspricht (Range 21-89\%). Diese 17 von insgesamt 75 Heimen im Bundesland, die sich auf 8 von 10 Bezirken im Bundesland Kärnten und 10 Trägerorganisationen verteilen, sind freiwillige Teilnehmer eines Projekts zur Versorgungsoptimierung der Autoren; die Befragung fand vor Beginn der Maßnahmensetzung statt. Ein Drittel der Befragten (31 \%) ist diplomiertes Pflegepersonal (also Pflegepersonen, die eine 3-jährige Ausbildung an einer Schule für Gesundheits- und Krankenpflege absolviert haben), $63 \%$ sind nichtdiplomiertes Pflegepersonal (51\% Pflegehelfer, $12 \%$ Altenfachbetreuer) und $5 \%$ der Befragten machten hierzu keine Angaben. Ein Drittel der Befragten (31\%) weist weniger als 5 Jah- re Berufserfahrung in der Altenpflege auf, $29 \%$ haben zwischen 5 und 10 Jahren Berufserfahrung und $35 \%$ sind mit mehr als 10 Jahren Berufserfahrung sehr erfahren in der Pflege von alten Menschen (5\% machten keine Angaben zur Berufserfahrung). Der Anteil von Personen mit einer Berufserfahrung von mehr als 10 Jahren ist in der Gruppe der diplomierten Pflegekräfte mit $43 \%$ größer als in der Gruppe der nichtdiplomierten Pflegekräfte mit $34 \%$ (mittlere Berufserfahrung: 33 vs. $29 \%$, geringe Berufserfahrung: 24 vs. $37 \%$ ).

\section{Methodik der Datenauswertung}

Da bislang keine Hinweise zur Toleranz fehlender Werte zum BPW veröffentlicht wurden, wurde ein konservativer Zugang gewählt. Pro Skala wurde maximal ein fehlender Wert toleriert. Bei der Wissensskala wurden fehlende Werte als falsche Lösung gewertet, bei der SWESkala wurden fehlende Werte durch den personenspezifischen Mittelwert ersetzt. Die deskriptivstatistische und inferenzstatistische Datenauswertung erfolgte in SPSS. Die Verteilung der BPW-SWE-Skala ist deutlich rechtsschief, aber auch die Werte der BPWWissensskala weichen signifikant von der Normalverteilung ab (Shapiro-WilkTest jeweils $p<0,001)$. Die Effekte der Berufsgruppe wurden mittels t-Tests für unabhängige Stichproben berechnet, da der t-Test sehr robust gegenüber Verletzungen der Normalverteilungsannahme ist. Für die Analyse der Effekte der ordinalskalierten Variable Berufserfahrung wurde der Jonckheere-Terpstra-Test, ein parameterfreies Verfahren, eingesetzt. Zusammenhänge zwischen Wissen und Selbstwirksamkeitserwartung wurden mittels Pearson-Korrelation überprüft. Das a-Niveau wurde auf $5 \%$ festgelegt.

\section{Ergebnisse}

\section{Wissen}

Von den 23 Fragen der BPW-Wissensskala wurden im Mittel 12,31 Fragen (SD 3, 144; 95 \%-KI: 11,9-12,6) richtig be- 


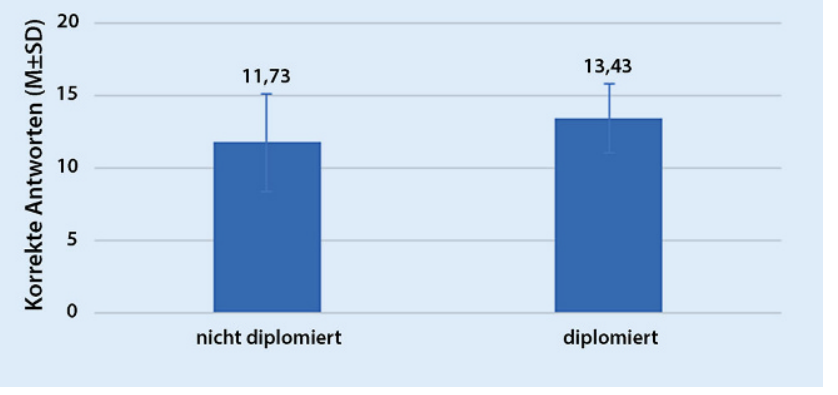

Abb. $3 \Delta$ Wissen im Bonner Palliativwissenstest nach Berufsgruppe. M Mittelwert; SD Standardabweichung

antwortet. • Abb. 1 zeigt den Prozentsatz richtiger Antworten auf Einzelitemebene. Im Durchschnitt wurden demnach $53,0 \%$ der Fragen korrekt beantwortet. Item 12 („Bewohnern mit lebensbedrohlichen Erkrankungen sollte immer die Wahrheit gesagt werden, damit sie sich auf ihren Tod vorbereiten können") weist eine sehr hohe Itemschwierigkeit auf. Die Items 18 („Sichtbare Rituale und Verabschiedungen beim Tod eines Bewohners sollten vermieden werden, um keine Unruhe zu erzeugen.“), 5 („Adjuvante Therapien (z. B. physikalische Maßnahmen) sind für die Schmerzbehandlung wichtig.“) und 15 („Kommunikationsfähigkeit kann erlernt werden.“) wurden von mehr als $80 \%$ der Befragten korrekt beantwortet.

\section{Selbstwirksamkeitserwartung}

Die SWE (BPW-SWE-Skala) ist sehr hoch ausgeprägt. Mit einem Mittelwert von $\mathrm{M}=3,31(\mathrm{SD}=0,414)$ liegt sie deutlich über dem theoretischen Skalenmittelwert. - Abb. 2 zeigt die deskriptive Auswertung auf Einzelitemebene, wobei der prozentuale Anteil an Befragten mit hoher SWE (stimmt eher, stimmt) dargestellt ist. Die SWE war demnach in Bezug auf die Items 6 („Ich denke, dass ich fähig bin, eine Vermittlung an Hospizdienste zu organisieren.“) und 12 („Ich denke, dass ich fähig bin, den Bewohnern die möglichen Nebenwirkungen der verschriebenen Medikamente zu erläutern.") vergleichsweise am geringsten ausgeprägt. Am höchsten war die SWE in Bezug auf die Minderung von Übelkeit ausgeprägt. Andererseits fühlen

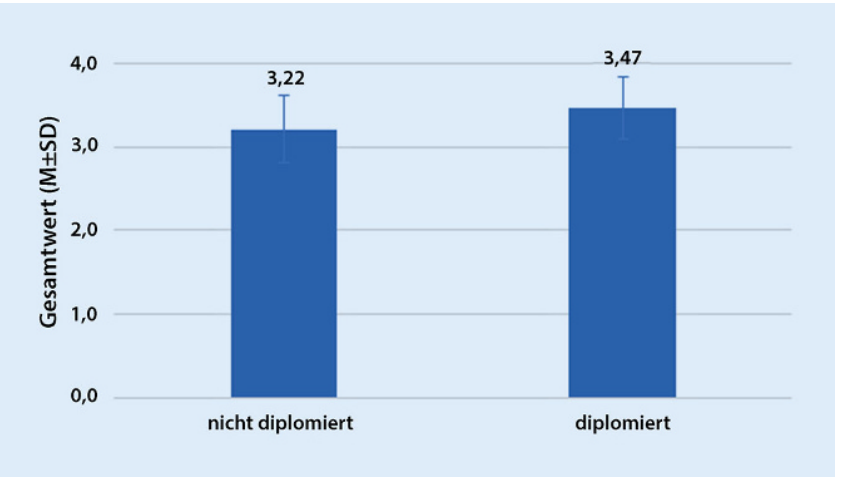

Abb. $4 \Delta$ Gesamtwert der Selbstwirksamkeitserwartung im Bonner Palliativwissenstest nach Berufsgruppe. M Mittelwert; SD Standardabweichung sich beispielsweise 80,5\% der Befragten in der Lage, einen Bewohner und seine Familie über die Palliativversorgung in der Einrichtung zu informieren.

\section{Effekte der Berufsgruppe}

Effekte der Berufsgruppe konnten in Bezug auf das Wissen und die SWE nachgewiesen werden. Diplomierte Pflegepersonen konnten im Schnitt rund eineinhalb Fragen mehr richtig beantworten als nichtdiplomierte Pflegepersonen $(\mathrm{t}[256,834]=-4,893, p<0,001$; - Abb. 3).

Diplomierte Pflegepersonen wiesen eine signifikant höhere SWE auf als nichtdiplomierte Pflegepersonen $(\mathrm{t}[304]=$ $-5,243, p<0,001 ; \bullet A b b .4)$.

\section{Effekte der Berufserfahrung}

Die Berufserfahrung hat keinen signifikanten Effekt auf das Wissen $(\mathrm{z}=0,332$; $p=0,740$; • Abb. 5).

Mit zunehmender Berufserfahrung steigt jedoch die SWE $(z=3,254 ; p=$ 0,001; • Abb. 6).

\section{Korrelation von Wissen und SWE}

Es zeigte sich kein Zusammenhang zwischen Wissen und Selbstwirksamkeitserwartung $(r=-0,043)$. Auch wenn man die Korrelationen getrennt nach Berufsgruppe oder Berufserfahrung berechnet, bleibt der extrem geringe Zusammenhang aufrecht (zwischen $r=0,018$ und $r=-0,131)$.

\section{Diskussion}

In der vorliegenden Studie beantworteten die Pflegekräfte im Durchschnitt etwas mehr als die Hälfte der Wissensfragen korrekt. Der Wissenstand ist trotz Unterschieden in der Ausbildung [17] durchaus vergleichbar mit den Ergebnissen einer Befragung deutscher Altenpflegekräfte. So wurden in der vorliegenden Studie durchschnittlich 53,0\% der Fragen korrekt beantwortet, in einer Stichprobe deutscher Pflegekräfte aus dem Altenpflegebereich $(n=130)$ lag der Anteil bei 54,7\% [21], in einer Expertenstichprobe $(n=23,[21,22])$ bei $80,3 \%$. Item 12 („Bewohnern mit lebensbedrohlichen Erkrankungen sollte immer die Wahrheit gesagt werden, damit sie sich auf ihren Tod vorbereiten können.") wies mit 18,8\% den geringsten Anteil korrekter Lösungen auf; mit einer Lösungswahrscheinlichkeit von 54,2\% war dieses Item auch in der Expertenstichprobe von Pfister et al. [21, 22] eines der schwierigsten. Diplomierte Pflegepersonen wiesen erwartungsgemäß ein signifikant höheres Wissen auf als nichtdiplomierte Pflegepersonen (vgl. [28]), wenngleich der Wissensvorsprung von im Schnitt eineinhalb Items eher gering ausfällt. Ein Effekt der Berufserfahrung auf das Wissen konnte jedoch nicht nachgewiesen werden.

Die SWE war in der vorliegenden Studie hoch ausgeprägt (deutlich über dem theoretischen Skalenmittelwert), wobei der SWE-Gesamtwert etwas höher im Vergleich zur deutschen Altenpflegestichprobe und deutlich unter jenem der Expertenstichprobe liegt [21, 22]. Auf 


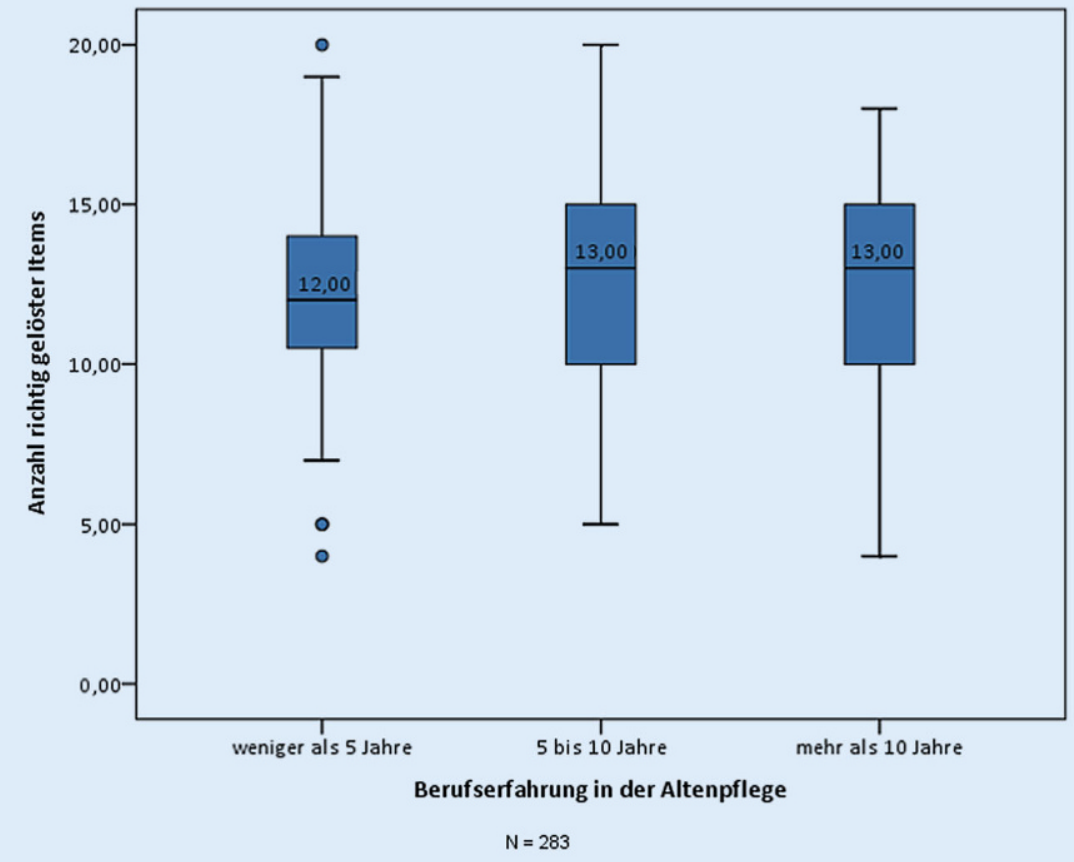

Abb. 5 ॥ Wissen im Bonner Palliativwissenstest nach Berufserfahrung

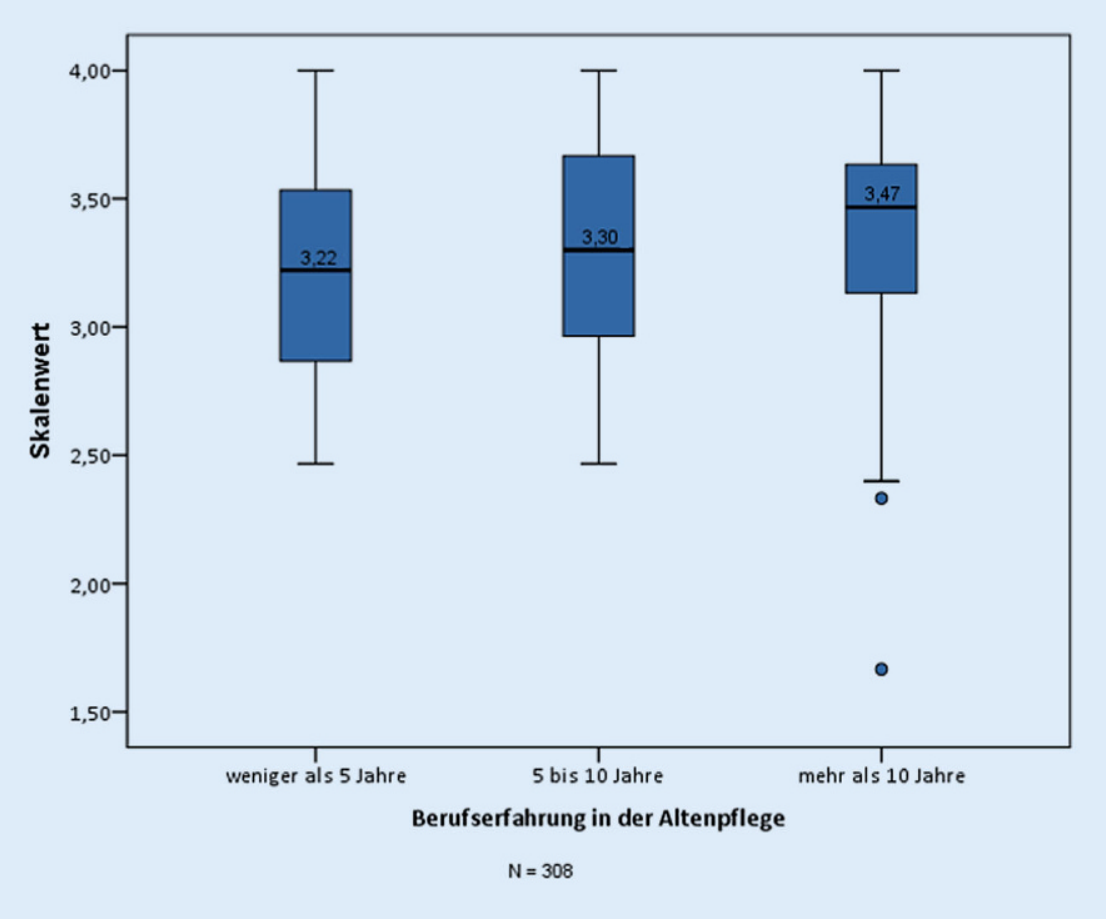

Abb. 6 - Selbstwirksamkeitserwartung im Bonner Palliativwissenstest nach Berufserfahrung

Einzelitemebene sind jedoch Unterschiede zu erkennen. Die eigene Kompetenz zur Minderung von Übelkeit bei Bewohnern schätzten 98,2\% der Befragten der vorliegenden Studie als hoch oder eher hoch ein, während dieser Anteil in der Studie aus Deutschland lediglich bei 77,7 \% lag. Auch in Bezug auf die Erfassung des Schmerzniveaus fühlten sich die Teilnehmer der vorliegenden Studie vergleichsweise kompetenter (96,4\% vs. 73,3\%). Die Einschätzung der eigenen
Kompetenz in Bezug auf das Erläutern von Medikamentennebenwirkungen als hoch oder eher hoch liegt mit $62,1 \%$ in einer ähnlichen Größenordnung wie in der deutschen Stichprobe. Nur $64 \%$ der Befragten fühlten sich (eher) kompetent, eine Vermittlung an Hospizdienste $\mathrm{zu}$ organisieren, während dieser Anteil bei deutschen Altenpflegekräften mit 73,8 \% höher war [21]. Diplomierte Pflegepersonen wiesen eine höhere SWE auf als nichtdiplomierte Pflegepersonen. Pflegepersonen mit längerer Berufserfahrung wiesen theoriekonform auch eine höhere SWE auf, während sich bei Pfister et al. [21] wider Erwarten eine negative Korrelation zwischen Berufserfahrung und Selbstwirksamkeitserwartung zeigte. Direkte Erfahrungen eigener Kompetenz stellen die stärkste Quelle der SWE dar $[1,2,27]$, mit zunehmender Berufserfahrung in der Altenpflege ist auch von einer Zunahme solcher SWE-stärkenden Erfahrungen auszugehen (vgl. [7]).

Wissen und SWE waren in der vorliegenden Studie nicht miteinander assoziiert, was vor dem Hintergrund der sozial-kognitiven Theorie von Bandura nicht überraschend ist $[1,2]$. So wiesen auch kanadische Pflegekräfte eine hohe Selbstwirksamkeit in Palliative Care auf trotz deutlicher Wissenslücken ([7], vgl. auch [21]). Einerseits stellt eine sehr hohe SWE ohne entsprechendes Wissen oder Kompetenzen zwar eine Überschätzung der eigenen Fähigkeiten dar [27], andererseits ist laut Bandura [1] gerade diese optimistisch erhöhte SWE durchaus von Vorteil, da sie dazu motiviert, (neue) Aufgaben in Angriff zu nehmen und Ziele mit Persistenz zu verfolgen. Diese Unterschiede in der SWE sind eine Erklärung dafür, dass Personen trotz gleichen Wissens oder gleicher Fähigkeiten unterschiedlich mit Herausforderungen und Stressoren umgehen. In einem Arbeitsumfeld wie der stationären Altenpflege, welches von Unsicherheit, Ungewissheit und zahlreichen Belastungen geprägt ist $[6,12]$, ist eine hohe Selbstwirksamkeit sicherlich eine notwendige Voraussetzung, um zu bestehen (vgl. dazu z. B. [26]).

Die Ergebnisse sprechen dafür, dass Maßnahmen zur Steigerung des Palliativwissens bei diplomiertem und nichtdiplomiertem Pflegepersonal in Alten- 
und Pflegeheimen erforderlich sind. Denn adäquates Wissen ist eine wichtige Voraussetzung für die angemessene Palliative-Care-Symptombehandlung [7], wodurch unter anderem belastende Krankenhaustransporte am Lebensende verhindert werden können [28]. Zur Umsetzung von HPC in Pflegeheimen liegen Richtlinien von Hospiz Österreich vor, die u.a. die (Weiter-)Entwicklung von Fort- und Weiterbildungsangeboten umfassen [10]. Diesen Richtlinien entsprechende Modellprojekte wurden bereits in mehreren Alten- und Pflegeheimen in Österreich umgesetzt [14]. Eine verstärkte Verankerung der Thematik Palliative Care in der Ausbildung von Pflegepersonen ist jedoch zusätzlich erforderlich [8, 18, 22]. Im Zuge der Implementierung etwaiger Maßnahmen zur Steigerung des Palliativwissens sind jedenfalls auch SWE-fördernde Elemente empfehlenswert, u. a. die Anerkennung bestehenden Wissens [7] und vor allem das Fördern von Erfolgserlebnissen [2]. Die vergleichsweise geringste SWE der Befragten in Bezug auf die Vermittlung zu Hospizdiensten spricht gerade vor dem Hintergrund des Modells der abgestuften Hospiz- und Palliativversorgung in Österreich [9] und der zentralen Rolle der mobilen Palliativteams in Kärnten [25] für die Notwendigkeit von Interventionen. Womöglich sind aber auch Unterschiede im Wording - in Österreich spricht man von mobilen Palliativteams oder Hospizteams und nicht von Hospizdiensten - für diesen Befund (mit-)verantwortlich. Fortbildungen in Palliative Care sollten Idealerweise berufsgruppenübergreifend angelegt sein [4], zumal auch die adäquate Versorgung von geriatrischen Palliativpatienten ein Hand-in-Hand-Arbeiten (zumindest) von Pflege und Medizin erfordert [9, 15, 24]. Schmerzassessment bei Bewohnern mit eingeschränkter Auskunftsfähigkeit aufgrund von Demenz muss dabei einen Schwerpunkt darstellen, da diese besonders häufig schmerztherapeutisch unterversorgt sind [3]. Schulungen sollten sich nicht auf reine Wissensvermittlung beschränken, sondern reflexive Elemente und gemeinsame Diskussionen sollten dabei Raum haben [18].
Die vorliegende Studie ist nicht frei von Limitationen. Auch wenn die Beteiligung an der Befragung durchaus hoch war, so ist ein Selektionsbias nicht auszuschließen. Möglicherweise haben also Pflegepersonen mit höherem Wissen vermehrt an der Befragung teilgenommen, sodass das vorhandene Wissen überschätzt wird. Auch das Messinstrument selbst könnte eine Limitation darstellen, da es bislang nur an 2 Stichproben $(n=130$ Altenpflegepersonen und $n=23$ Experten, $[21,22]$ ) erprobt wurde. Um Personenunterschiede abzubilden werden gemeinhin Items mit einer Schwierigkeit zwischen 20 und $80 \%$ empfohlen, sodass einige Items des BPW möglicherweise zu leicht sind [5]. Die Dimensionalität wurde bislang nicht geprüft. Somit ist unklar, ob die additive Zusammenfassung zu einem Gesamtwert überhaupt zulässig ist [5]. Dennoch liefert die vorliegende Studie eine erste Evidenzbasis zu Wissen und SWE von Pflegekräften in der stationären Langzeitpflege in Österreich im Umgang mit Palliativpatienten. Künftige Studien sollten genauer analysieren, inwiefern palliativbezogenes Wissen und SWE tatsächlich entsprechend kompetentes pflegerisches Verhalten nach sich ziehen. Da Personen trotz geringem Wissen bisweilen eine hohe SWE aufweisen, stellt sich die Frage, inwiefern die Erfolgserlebnisse aus der Praxis - die stärkste Quelle der SWE - tatsächlich „echten“ Erfolgen guter Palliativpflege entsprechen oder nur eine (Fehl-)Beurteilung des eigenen Erfolgs aufgrund mangelnden Wissens darstellen. Dem geringen Wissensunterschied zwischen diplomiertem und nichtdiplomiertem Pflegepersonal bzw. den Ursachen hierfür sollte ebenfalls in weiteren Untersuchungen auf den Grund gegangen werden.

\section{Fazit für die Praxis}

Pflegekräften in Alten- und Pflegeheimen in Kärnten (Österreich) schätzten ihre Kompetenzen in der PalliativeCare-Versorgung hoch ein. Gleichzeitig sind ähnlich wie bei deutschen Pflegekräften [21, 22] deutliche Wissenslücken festzustellen. Es besteht demnach Handlungsbedarf in der
Schulung von Pflegekräften im Pflegeheimbereich, da sie zunehmend mit Palliativpatienten konfrontiert sind. Schulungen sollten reflexive und diskursive Elemente enthalten [18] und die Schmerzerkennung bei Bewohnern mit Demenz berücksichtigen [3]. Eine verstärkte Zusammenarbeit von Pflegeheimen mit den in Kärnten bereits gut etablierten mobilen Palliativteams ist zu empfehlen, zumal aktuelle Befunde belegen, dass durch Palliative-CareKonsile in Pflegeheimen belastende Krankenhaustransporte am Lebensende reduziert werden können [16]. Entsprechendes Wissen, Kompetenzen und SWE aufseiten des Pflegepersonals sind eine wichtige Grundvoraussetzung für die Implementierung von HPC in Pflegeheimen. Selbstverständlich müssen dabei auch auf Organisationsebene die entsprechenden Rahmenbedingungen geschaffen werden (vgl. [10]). Fort- und Weiterbildungen für das diplomierte und nichtdiplomierte Pflegepersonal sind zwar eine notwendige Voraussetzung zur Verbesserung von Palliative Care in Pflegeheimen, jedoch kann eine nachhaltige Verankerung von HPC in Pflegeheimen nur gelingen, wenn durch entsprechende Prozesse der Organisationsentwicklung eine "Kultur des Lebens und Sterbens" implementiert wird [9, $18,23]$.

\section{Korrespondenzadresse}

FH-Prof. Mag. Dr. rer. nat. O. Kada

Studiengang Gesundheits- und Pflegemanagement, FH Kärnten

Hauptplatz 12, 9560 Feldkirchen, Österreich

o.kada@fh-kaernten.at

Open access funding provided by Carinthia University of Applied Sciences (CUAS).

\section{Einhaltung ethischer Richtlinien}

Interessenkonflikt. O. Kada, H. Janig, G. Pinter, K. Cernic und R. Likar geben an, dass kein Interessenkonflikt besteht.

Alle beschriebenen Untersuchungen am Menschen wurden mit Zustimmung der zuständigen EthikKommission, im Einklang mit nationalem Recht sowie gemäß der Deklaration von Helsinki von 1975 (in der aktuellen, überarbeiteten Fassung) durchgeführt. 
Open Access. Dieser Artikel wird unter der Creative Commons Namensnennung 4.0 International Lizenz (http://creativecommons.org/licenses/by/4.0/deed. de) veröffentlicht, welche die Nutzung, Vervielfältigung, Bearbeitung, Verbreitung und Wiedergabe in jeglichem Medium und Format erlaubt, sofern Sie den/die ursprünglichen Autor(en) und die Quelle ordnungsgemäßnennen, einen Link zur Creative Commons Lizenz beifügen und angeben, ob Änderungen vorgenommen wurden.

\section{Literatur}

1. Bandura A (1997) Self-efficacy. The exercise of control. W.H. Freeman and Company, New York

2. Bandura A (2012) On the functional properties of perceived self-efficacy revisited. J Manage 38(1):9-44. doi:10.1177/0149206311410606

3. Bauer U, Pitzer S, Schreier MM, Osterbrink J, Alzner $\mathrm{R}$, Iglseder B (2016) Pain treatment for nursing home residents differs according to cognitive state - a cross-sectional study. BMC Geriatr 16:124. doi:10.1186/s12877-016-0295-1

4. Becker G, Momm F, Deibert P, Xander C, Gigl A, Wagner B, Baumgartner J (2010) Planning training seminars in palliative care: a cross-sectional survey on the preferences of general practitioners and nurses in Austria. BMC Med Educ 10:43. doi:10. 1186/1472-6920-10-43

5. Bortz J, Döring N (2006) Forschungsmethoden und Evaluation für Human- und Sozialwissenschaftler. Springer, Berlin

6. Brause M, Kleina T, Horn A, Schaeffer D (2015) Burnout-Risiko in der stationären Langzeitversorgung. Ressourcen und Belastungen von Pflege- und Betreuungskräften. Präv Gesundheitsf 10(1):41-48. doi:10.1007/s11553-014-0469-7

7. Brazil K, Brink P, Kaasalainen S, Kelly ML, McAiney $C$ (2012) Knowledge and perceived competence among nurses caring for the dying in long-term care homes. Int J Palliat Nurs 8(2):77-83. doi:10. 12968/ijpn.2012.18.2.77

8. Brunner E, Kada O (2010) Professionalisation of gerontological nursing - the development of an international online gerontological master degree programme. Nurse Educ Today 30(8):779-783. doi:10.1016/j.nedt.2010.03.001

9. GÖG (2014) Abgestufte Hospiz- und Palliativversorgung für Erwachsene. Aktualisierung 2014 (2. Auflage). http://www.bmg. gv.at/cms/home/attachments/3/6/7/CH1071/ CMS1103710970340/broschuere_hospiz-_und palliativversorgung_1_12_2014.pdf.Zugegriffen: 2. September 2015

10. Hospiz Österreich (2009) Richtlinien Umsetzung und Entwicklung von Hospiz und Palliative Care im Pflegeheim. http://www.hospiz.at/pdf dl/Richtlinien_hpcph_2010.pdf. Zugegriffen: 2 . September 2015

11. Janig H, Kada O, Neuwersch S, Likar R (2015) Wie sehen Notärzte ihre Einsätze in Pflegeheimen? Eine explorative Studie. Pflege Z68(8):494-499

12. Kada O, Janig H (2016) Mixed methods in action: the dilemma of nursing home residents relocated to hospital.In:SAGE research methods cases. SAGE, London

13. Kada O, LikarR, Pipam W, Miklautz M, Janig H (2007) Tumorschmerz aus Sicht der Pflege - Befunde aus Österreich.Pflege Z60(11):626-630

14. Kränzl-Nagl R, Nußbaumer B (2012) Bestandsaufnahme zu Hospiz und Palliative Care in Österreichs Alten-und Pflegeheimen. Endbericht Kurzfassung.
http://www.hospiz.at/pdf_dl/HP_Endbericht Kurzversion_final.pdf.Zugegriffen:21. März 2016

15. Lukas A, Mayer B, Odner G, Bernabei R, Denkinger MD (2015) Schmerztherapie im deutschen Pflegeeinrichtungen im europäischen Vergleich. Ergebnisse der SHELTER-Studie. Schmerz 29(4):411-421. doi:10.1007/s00482-015-0004-6

16. Miller SC, Dahal R, Lima JC, Intrator O, Martin E, Bull J, Hanson LC (2016) Palliative care consultations in nursing homes and end-of-life hospitalizations. J Pain Symptom Manage. doi:10. 1016/j.jpainsymman.2016.05.017.

17. Mir E, Kada O, Brunkel H, Kohlmann E, Kohlmann CW (2016) Wie nehmen Auszubildende der Altenpflege die Arbeits- und Organisationsstrukturen in der Praxis wahr? Erfahrungen mit einem arbeitspsychologischen Screeningverfahren. HeilberufeScience. doi:10.1007/s16024-016 0263-x

18. Müller D (2016) Der Einfluss des Personals auf die Lebensqualität und Sterbekultur in Pflegeheimen. In: Kojer M, Schmidl M (Hrsg) Demenz und Palliative Geriatrie in der Praxis. Heilsame Betreuung unheilbar demenzkranker Menschen. Springer, Wien, S215-223

19. Nestler N, Gnass I, Schuler M (2015) Schmerz in Einrichtungen der stationären Altenpflege. Schmerz 29(1):131-141. doi:10.1007/s00482014-1484-5

20. Onder G, Carpenter I, Finne-Soveri H, Gindin J, Frijters D, Henrard JC, Nikolaus T, Topinkova E, Tosato M, Liperoti R, Landi F, Bernabei R, SHELTER project (2012) Assessment of nursing home residents in Europe: the Services and Health for Elderly in Long TERm care (SHELTER) study. BMC Health Serv Res 12:5. doi:10.1186/1472-6963-125

21. Pfister D, Markett $S$, Müller M, Müller $S$, Grützner F, Rolke R, Kern M, Schmidt-WolfG, Radbruch L (2013) German nursing home professionals' knowledge and specific self-efficacy related to palliative care. J Palliat Med 16(7):794-798. doi:10.1089/jpm.2012. 0586

22. Pfister D, Müller M, Müller S, Kern M, Rolke R, Radbruch L (2011) Validierung des Bonner Palliativwissenstests (BPW). Schmerz 25(6):643-653. doi:10.1007/s00482-011-1111-7

23. Pleschberger S (2006) Palliative Care in Pflegeheimen - Forschungsstand und Entwicklungsperspektiven. Z Gerontol Geriatr 39:376-381. doi:10. 1007/s00391-006-0351-0

24. Sandgathe Husebø B, Husebø S (2001) Palliativmedizin - auch im hohen Alter? Schmerz 15(5):350-356. doi:10.1007/s004820170009

25. Schippinger W, Likar R, Traar B, Pinter G, Janig H (2013) Palliativmedizin - Sterben und Tod älterer Menschen. In: Pinter G, Likar R, Schippinger W, Janig H, Kada O, Cernic K (Hrsg) Geriatrische Notfallversorgung. Strategien und Konzepte. Springer, Wien, S129-149

26. Schwarzer R, Hallum S (2008) Perceived teacher self-efficacy as a predictor of job stress and burnout: mediation analyses. Appl Psychol 57(Supplement 1):152-171

27. Schwarzer R, Jerusalem M (2002) Das Konzept der Selbstwirksamkeit. Z Pädag 44:28-53 (Beiheft: Selbstwirksamkeit und Motivationsprozesse in Bildungsinstitutionen)

28. Unroe KT, Cagle JG, Lane KA, Callahan CM, Miller SC (2015) Nursing home staff palliative care knowledge and practices: results of a large survey of frontline workers. J Pain Symptom Manage 50(5):622-629. doi:10.1016/j.jpainsymman.2015. 06.006
Hier steht eine Anzeige. 袋 Springer 